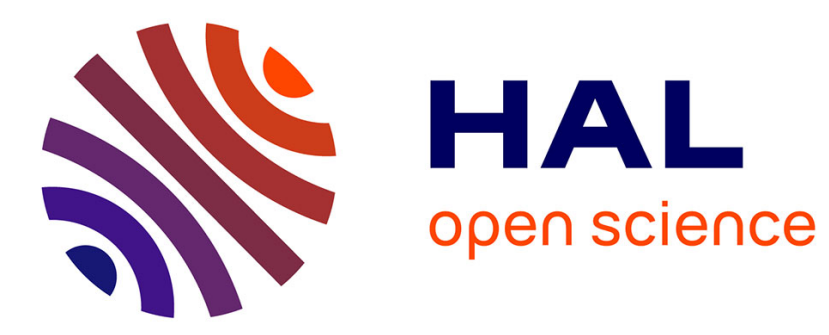

\title{
Observation of quasi-particle distribution in non-equilibrium superconductors
}

\author{
B. Lambert, D. Salin, J. Joffrin
}

\section{To cite this version:}

B. Lambert, D. Salin, J. Joffrin. Observation of quasi-particle distribution in nonequilibrium superconductors. Journal de Physique Lettres, 1977, 38 (17), pp.343-345. 10.1051/jphyslet:019770038017034300 . jpa-00231392

\section{HAL Id: jpa-00231392 https://hal.science/jpa-00231392}

Submitted on 1 Jan 1977

HAL is a multi-disciplinary open access archive for the deposit and dissemination of scientific research documents, whether they are published or not. The documents may come from teaching and research institutions in France or abroad, or from public or private research centers.
L'archive ouverte pluridisciplinaire HAL, est destinée au dépôt et à la diffusion de documents scientifiques de niveau recherche, publiés ou non, émanant des établissements d'enseignement et de recherche français ou étrangers, des laboratoires publics ou privés. 


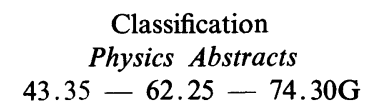

\title{
OBSERVATION OF QUASI-PARTICLE DISTRIBUTION IN NON-EQUILIBRIUM SUPERCONDUCTORS
}

\author{
B. LAMBERT, D. SALIN \\ Laboratoire d'Ultrasons (*), Université Pierre-et-Marie-Curie, \\ Tour 13, 4 place Jussieu, 75230 Paris Cedex 05, France \\ and

\section{J. JOFFRIN} \\ Institut Laüe-Langevin, $156 \mathrm{X}$, Centre de tri, 38042 Grenoble Cedex (France) \\ (Reçu le 31 mai 1977, accepté le 29 juillet 1977)

\begin{abstract}
Résumé. - La mesure de l'absorption d'ondes acoustiques dans des films minces d'étain excités au moyen d'un laser pulsé nous a permis d'observer directement la distribution des quasi-particules dans un supraconducteur porté hors d'équilibre par excitation lumineuse. Les résultats sont en accord avec le modèle thermique modifié.
\end{abstract}

Abstract. - By measuring the absorption of acoustic waves in thin-films of tin irradiated by laser pulses, we have been able to observe directly the quasi-particle distribution in a superconductor driven out of equilibrium by the light intensity. The results support the modified heating model.

Recently, considerable attention has been paid to properties of superconductors driven out of equilibrium by illuminating thin films or by exposing them to microwaves, phonons or injected quasi-particles [1].

Owen and Scalapino [2] first developed a model where the quasi-particles (q.p.) were treated as a Fermi gas at the temperature $T$ of the lattice, assuming that the recombination time for q.p. to form pairs is much larger than the characteristic time for their thermalization to the lattice; thus, the system is in thermal equilibrium but not in chemical equilibrium. The metastable distribution function for the q.p. is

$$
f(E)=\frac{1}{1+\exp \beta\left(E-\mu^{*}\right)}
$$

where $\mu^{*}$ is the chemical potential. The model (the so-called $\mu^{*}$ model) is able to give the energy gap $\Delta$ as a function of $n$, the excess q.p. number in units of $4 N(\mathrm{o}) \Delta_{0} ; N(\mathrm{o})$ is the single spin density of states at the Fermi level and $\Delta_{0}$ the equilibrium energy gap at $T=0 \quad\left(\mu^{*}=0\right.$ for $\left.n=0\right)$. The most striking prediction of this model is a first-order transition to

$\left({ }^{*}\right)$ Associated with the Centre National de la Recherche Scientifique. the normal state at a value of $n \simeq 0.15$ which occurs as long as the reduced temperature

$$
t=\frac{T}{T_{\mathrm{c}}} \ll 1 .
$$

For small $n$ the comparison between experimental ard theoretical results is quite satisfactory.

However recent experiments, in which the superconductor is illuminated by laser pulses, show that the first-order transition is not observed in either resistivity or microwave reflectivity measurements. Parker [3] has proposed a modified model in which the q.p. are in thermal and chemical equilibrium; phonons with energies greater than $2 \Delta$ are assumed to be out of equilibrium and may be described by an effective temperature $T^{*}$ which is different from the temperature of the lattice ( $T^{*}$-model). It is possible to calculate $T^{*}(n)$ simply by assuming that these phonons are described by a thermal distribution; all the subsequent properties are finally described by considering a BCS superconductor at a temperature $T^{*}$. In particular the transition to the normal state is of second order. It is important to note that the curves $\Delta(n)$ corresponding to the two distribution functions are nearly similar for $n \leqslant 0.15$.

The life-time for near equilibrium states has been obtained from a solution of the kinetic equation, 
including a term for the decay of phonons which escape from the film [4]. Recently Chang and Scalapino [5] have considered the energy distribution of quasi-particles and phonons for a variety of nonequilibrium situations in the case of weak external drives. Their results indicate that the distributions are dependent on the mode of excitations ; under certain conditions, which are in particular those of the laser experiments, the distributions are quite well approximated by the $T^{*}$-model. Moreover experimental results [6] on superconducting $\mathrm{Sn}$ excited by quasiparticle injection are in agreement with the theory of Owen and Scalapino. In view of these recents results, it seems that excitations formed by quasiparticles of energy slightly above the gap are well approximated by the Owen-Scalapino model; in contrast, quasi-particles generated by optical excitation (high energy excitation) may be described by the Parker model.

However the interpretation of most of the experiments done so far requires à lengthy treatment of the distribution function provided by the various models. It is the aim of the experiment reported below to try to reach the distribution function directly; bearing in mind the well-known result of Bömmel [7], who measured the acoustic attenuation as a function of $T / T_{\mathrm{c}}$ in the superconducting state and who proved that the acoustic attenuation varies as the FermiDirac distribution function just above the gap, the result predicted by B.C.S. theory, we have measured the attenuation of acoustic waves in a thin film of tin exposed to laser pulses. As in the B.C.S. case, the attenuation is simply related to the distribution function of the q.p. above the gap; the theory predicts that the ratio of the attenuation in the superconducting state to that of the normal state is

$$
\frac{\alpha_{\mathrm{s}}}{\alpha_{\mathrm{N}}}=2 f(\Delta) \text { for } \hbar \omega \ll \Delta
$$

where $f(\Delta)$ is the Fermi-Dirac function for $E=\Delta$. Thus, the measurement of this ratio enables the different models to be distinguished. In the case of the $\mu^{*}$-model we obtain :

$$
\frac{\alpha_{\mathrm{S}}}{\alpha_{\mathrm{N}}}=\frac{2}{1+\exp \beta\left(\Delta-\mu^{*}\right)} .
$$

In the other case $\left(T^{*}\right.$-model $)$ the ratio $\alpha_{S} / \alpha_{N}$ is given by

$$
\frac{\alpha_{\mathrm{S}}}{\alpha_{\mathrm{N}}}=\frac{2}{1+\exp \beta^{*} \Delta\left(T^{*}\right)} .
$$

To illustrate these results, we have plotted in figure 1 the value $\alpha_{\mathrm{S}} / \alpha_{\mathrm{N}}$ for $t=T / T_{\mathrm{c}}=0.36$ corresponding to the two models : the figure shows that the ultrasonic measurements are able to test the validity of the models. This figure may be compared with the curves of $\Delta(n)$ (see for example Fig. 3 of ref. [3]).

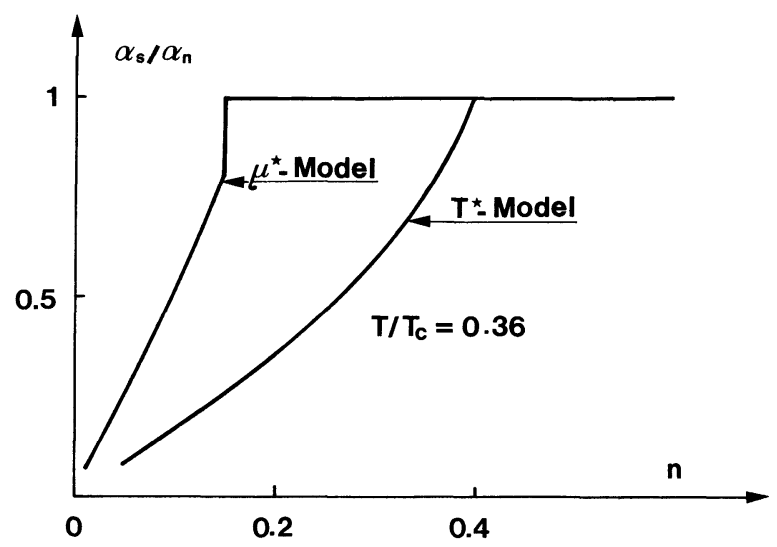

FIG. 1. - The acoustic attenuation $\alpha_{S} / \alpha_{N}$ is plotted as a function of $n$ (see text) for the $T^{*}$-model and the $\mu^{*}$-model. The reduced temperature $t=T / T_{\mathrm{c}}=0.36$.

[L'atténuation acoustique $\alpha_{\mathrm{S}} / \alpha_{\mathrm{N}}$ est portée en fonction de $n$ (voir le texte) dans les cas du modèle $T^{*}$ et du modèle $\mu^{*}$. La valeur de la température réduite est $t=T / T_{\mathrm{c}}=0,36$.]

The experiment is performed with a film of tin deposited on a A.C. quartz substrate. The film thickness, $d=3000 \AA$, is approximately adjusted to match a mechanical resonance condition $d=3 / 2 \lambda$ for a transversely polarized acoustic wave at $9 \mathrm{GHz}$ $(\lambda \simeq 2000 \AA$ in tin). Transverse waves are used in preference to longitudinal waves in order to increase the observed effect. The value of $\alpha_{N}$ is measured by applying a magnetic field to the sample : the acoustic signal difference between $H=0$ and $H>H_{\text {critical }}$ is a measurement of $\alpha_{\mathrm{N}}$; at low temperature (e.g. $T / T_{\mathrm{c}}=0.36$ with $T_{\mathrm{c}}=3.72 \mathrm{~K}$ for $\mathrm{Sn}$ ) it is found to be $1.7 \pm 0.1 \mathrm{~dB} . \alpha_{\mathrm{S}}$ is measured at the same temperature as a function of the optical pulse intensity $I$; the acoustic signal difference between $I=0$ and $I$ is a measurement of $\alpha_{\mathrm{s}}$. The film, the quartz substrate and the microwave cavity used to produce the acoustic, wave, are all immersed in liquid helium maintained at $1.3 \mathrm{~K}$.

We use a $Q$-switched YAG/Nd laser delivering adjustable pulses of a few hundred nanoseconds. The acoustic pulse length is of the same order of magnitude and is synchronized to the laser pulse. By varying the delay between the two pulses, it is possible to excite the tin film both by the light and by the acoustic wave simultaneously (in which case we measure $\alpha_{\mathbf{s}}$ ) or to illuminate the film either before or after it resonates acoustically; by so doing, it is possible to correct the data for small spurious effects and to be sure that heating does not obscure the signal.

We plot $\alpha_{S} / \alpha_{N}$ as a function of the light intensity $I$ in figure 2. For $I$ larger than a value $I_{c}$, the echo reaches a plateau. By varying the intensity we have been able to approach close to the value $I_{\mathrm{c}}$; we have observed no discontinuity in the amplitude of the signal when $I$ reach the transition region. For this reason, we have compared our results with the $T^{*}$-model. Figure 2 presents a typical run for a given laser intensity. 


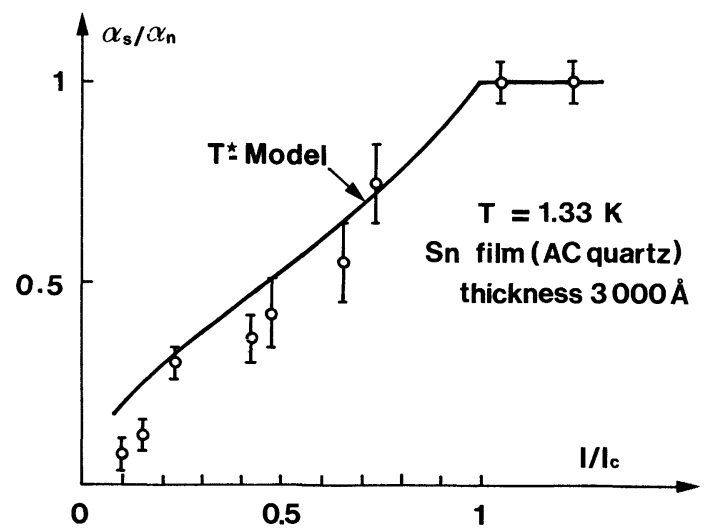

FIG. 2. - The points with error bars correspond to experimental results; $\alpha_{\mathrm{S}} / \alpha_{\mathrm{N}}$ is plotted as a function of the reduced light intensity $I / I_{\mathrm{c}}$. The curve corresponds to the $T^{*}$-model [3].

[Les points avec les barres d'erreur correspondent aux résultats expérimentaux; $\alpha_{\mathrm{S}} / \alpha_{\mathrm{N}}$ est porté en fonction de l'intensité lumineuse réduite $I / I_{\mathrm{c}}$. La courbe correspond au modèle $T^{*}[3]$.]

When $I>I_{\mathrm{c}}$ the effect remains constant and gives an attenuation of $1.7 \mathrm{~dB}$, that is, the film is normal. The error bars in figure 2 take into account both uncertainty due to the ultrasonic experiment and the statistical variation which was observed from laser pulse to pulse. The comparison between the experiment results and the calculated curve shows the good validity of the $T^{*}$-model, even if there remains a discrepancy at low powers.

Several numerical comparisons may be made. The amplitude of our effect is roughly $2 \mathrm{~dB}$, but the metallic film resonates with a mechanical $Q$ factor; with a thickness of $3000 \AA$, our effect would correspond to an attenuation per centimeter of

$$
\alpha_{\mathrm{dB} / \mathrm{cm}}=\frac{2}{3 \times 10^{-5} \cdot Q} \simeq \frac{10^{5}}{Q} .
$$

By comparing with other acoustic experiments at the same frequency [8] which give approximately a few thousands $\mathrm{dB} / \mathrm{cm}$, we find that $Q$ is of the order of 50 ; this is very reasonable in comparison with experiments performed on metallic films at the same frequency [9].
By varying the delay between the laser and the acoustic pulse it could be hoped to observe the recombination time $\tau_{\text {eff. }}$ of the q.p. Unfortunately our pulse duration is too long (a few hundred nanoseconds); it was shown experimentally [10] that $\tau_{\text {eff. }}$ is of the order of 100 nanoseconds at $1.3 \mathrm{~K}$ and that it rapidly decreases as $T$ increases. However it would be interesting to perform experiments with the particular aim of observing the parameters which govern the relaxation time : temperature and bottleneck effects.

Finally we try to calculate the critical intensity $I_{\mathrm{c}}$ and compare it with our measured value of about $500 \mathrm{~W} / \mathrm{cm}^{2}$ (only a fraction of which is absorbed by the tin film). In fact, there is a large uncertainty in the measurement of the absolute value of the flux reaching the sample; there is also some uncertainty in determining the photon efficiency since the number of q.p. created by each of them is not well known. If we take the number $r$ of quasi-particles produced per photon to be equal to 200 [11] and if we use the same numerical constants as S. B. Kaplan et al. [4] we find a value of $n_{\mathrm{c}}$ of the order of 1 . This is larger than the value required by the modified model $\left(n_{\mathrm{c}}=0.4\right)$ but in view of the experimental difficulties the agreement is satisfactory.

In conclusion, we have observed directly the q.p. distribution above the gap and it appears that our measurements support the $T^{*}$-model even though there remains some discrepancy. It has to be kept in mind that the Parker model involves the simplest possible assumption about the phonons of energy greater than $2 \Delta$. Thus, this model can only be used as a frame of reference for discussing, comparing and interpreting the experimental data; the situation appears to be nearly the same for the Owen-Scalapino model. On the other hand, the method has proved to be efficient and could very well be extended to other different type of excitations, such as quasi-particle injection.

Acknowledgments. - We wish to thank P. Levinson for providing us with the metallic films evaporated on the quartz substrates.

\section{References}

[1] See for example a recent review by LANGenberg, D. N., Proceeding of the 14th International Conference on Low Temperature Physics, Finland, 1975. Edited by M. Krusius and M. Vuorio (North Holland, Amsterdam) 1975.

[2] OWen, C. S. and Scalapino, D. J., Phys. Rev. Lett. 28 (1972) 1559.

[3] PARKer, W. H., Phys. Rev. B 12 (1975) 3667.

[4] Kaplan, S. B., Chi, C. C., Langenberg, D. N., Chang, J. J., Jafarey, S. and Scalapino, D. J., Phys. Rev. B 14 (1976) 4854.
[5] Chang, J. J. and Scalapino, D. J., Phys. Rev. B 15 (1977) 2651. [6] Fuchs, J., EPPERLEIn, P. W., Welte, M. and EisenMenger, W., Phys. Rev. Lett. 38 (1977) 919.

[7] BÖMmel, H. E., Phys. Rev. 96 (1954) 220.

[8] Bearzatto, C., Joffrin, J. and Levelut, A., Phys. Lett. A 33 (1970) 473.

[9] Split, G., Z. Phys. 225 (1969) 60.

[10] For a discussion of the experimental results, see [4].

[11] Schuller, I. and Gray, K. E., Phys. Rev. B 14 (1976) 4213. 